\title{
Tazarotene as alternative topical treatment for onychomycosis
}

\author{
This article was published in the following Dove Press journal: \\ Drug Design, Development and Therapy \\ 16 February 2015 \\ Number of times this article has been viewed
}

\author{
Elena Campione' \\ Evelin Jasmine Paternò ${ }^{2}$ \\ Gaetana Costanza ${ }^{2,3}$ \\ Laura Diluvio' \\ Isabella Carboni' \\ Daniele Marino ${ }^{4}$ \\ Cartesio Favalli ${ }^{4}$ \\ Sergio Chimenti' \\ Luca Bianchi' \\ Augusto Orlandi ${ }^{2,3}$ \\ 'Department of Dermatology, \\ ${ }^{2}$ Department of Biomedicine \\ and Prevention, ${ }^{3}$ Department \\ of Anatomic Pathology, Policlinic Tor \\ Vergata, ${ }^{4}$ Department of Microbiology, \\ University of Rome Tor Vergata, Rome, \\ Italy
}

Correspondence: Elena Campione Department of Dermatology, University of Rome Tor Vergata, Viale Oxford 8I, Rome 00133, Italy

Tel +390620900252

Fax +390620902742

Email campioneelena@hotmail.com
Background: Distal and lateral onychomycoses are the most frequent forms of onychomycosis, causing subungual hyperkeratosis that usually limits local penetration of antimycotic drugs. Tazarotene exerts anti-inflammatory and immune-modulating activities toward both infective agents and damaged keratinocytes. Given the well-documented efficacy of tazarotene on hyperkeratotic nail psoriasis, we investigated its therapeutic use in onychomycosis.

Patients and methods: We designed a preliminary open clinical trial in patients affected by distal and lateral subungual onychomycosis of the toenails and verified the fungistatic activity of tazarotene in vitro. Fifteen patients were treated with topical tazarotene $0.1 \%$ gel once per day for 12 weeks. Mycological cultures and potassium hydroxide stains of nail samples were performed at the beginning and at the end of the study. Treatment was considered effective when clinical healing and negative mycological culture were obtained. Onycholysis, nail bed discoloration, and subungual hyperkeratosis were measured using standardized methodologies and analyzed by means of Mann-Whitney test and analysis of variance. Fungistatic activity of tazarotene was evaluated by disk diffusion assay.

Results: Six patients (40\%) reached a mycological cure on target nail samples already after 4 weeks of treatment. Complete clinical healing and negative cultures were reached in all patients at week 12, with a significant improvement of all clinical parameters of the infected nails. Disk diffusion assay after 48 hours of incubation with tazarotene solution showed a central area of inhibition in all examined fungal cultures.

Conclusion: Our results documented a good clinical outcome using topical tazarotene $0.1 \%$ gel in distal and lateral subungual onychomycosis and its fungistatic activity of tazarotene in vitro. The majority of patients appeared cured at a 6-month follow-up. The efficacy and safety of tazarotene must be confirmed on a larger number of patients, although already documented in nail psoriasis patients often affected by onychomycosis.

Keywords: antiproliferative agent, retinoids, fungistatic activity

\section{Introduction}

Onychomycosis is a chronic fungal infection of the nail, characterized by nail discoloration, thickening, and defect. The involved pathogens are dermatophytes, yeasts (Candida), and nondermatophytes. ${ }^{1}$ Dermatophytes such as Trichophyton rubrum and Trichophyton mentagrophytes are the most common agents of onychomycosis, accounting for $50 \%-90 \%$ of cases, with prevalence of the infection higher in toenails than in fingernails. ${ }^{2}$ Candida and nondermatophytic molds are frequent in countries with hot and humid climate. ${ }^{3}$ If untreated, nail fungal infections can extend to other nails and potentially cause further complications, especially in at-risk patients. Aging and reduced peripheral circulation are among the major risk factors for onychomycosis; diabetes weakens the immune function, favoring slow nail growth, thickening, and susceptibility to infections and nail injury. ${ }^{4-7}$ Onychomycosis sometimes is associated with nail psoriasis, 
favored by abnormal capillary units and onychodystrophy. ${ }^{8}$ Differential diagnoses of onychomycosis include chronic nail eczema, bacterial infections, traumatic onychodystrophies, pachyonychia congenita, nail bed tumors, yellow nail syndrome, and idiopathic onycholysis. ${ }^{9}$ The treatment of onychomycosis is sometimes difficult because the predisposing factors are not influenced by therapy. ${ }^{10,11}$ Onychomycosis is usually treated with topical or systemic antifungals. ${ }^{12,13}$ Local treatment requires strong patient adherence because the treatment time is generally long; moreover, onychomycosis-related subungueal hyperkeratosis is generally thick and this limits the penetration of the antimycotic drug. Consequently, long-term treatments can discourage or induce stopping of the therapy before healing is achieved. The Cochrane meta-analysis of Crawford et al ${ }^{14}$ reports limited success in the treatment of onychomycosis with topical agents. Although many therapeutic options are available, new drugs with a greater safety and relatively rapid efficacy are required. One of the most used topical drug for nail psoriasis is tazarotene. ${ }^{15,16}$ Its efficacy is classically related to its immunomodulating properties and anti-inflammatory activity, with consequent inhibition of the infective process. ${ }^{17}$ Tazarotene is also successfully used for acne treatment, given its anti-inflammatory and immunemodulating effectiveness that protect keratinocytes from infection by Propionibacterium acnes. ${ }^{17}$ Epidermal penetration of tazarotene depends on its ability to reduce hyperkeratinization, favoring keratinocyte differentiation, as also documented by the reduction of expression of hyperproliferation-associated keratins 6 and $16 .{ }^{18}$ The latter appear dysregulated in genetic diseases, such as pachyonychia congenita and focal palmoplantar keratoderma. ${ }^{18}$ As a matter of fact, fungal infections could exacerbate or predispose an individual to pachyonychia congenita. ${ }^{19,20}$ In light of our previous studies reporting the clinical efficacy and the simplicity of the use of tazarotene in the nails of psoriatic patients, ${ }^{15,16}$ we investigated its effect on distal and lateral subungual onychomycosis in an open preliminary study of immunocompetent patients and its fungistatic activity in vitro.

\section{Patients and methods}

We report an observational open clinical trial in 15 patients affected by onychomycosis of the toenail treated with topical tazarotene (Zorac/Tazorac ${ }^{\circledR}$; Allergan Inc, Irvine, CA, USA) $0.1 \%$ gel in an off-label use. The enrolled patients were of both sexes and aged between 14 years and 70 years (Table 1). Patients eligible were those affected by distal and lateral subungual onychomycosis without associated skin or systemic diseases, naïve or in washout for other treatments. Exclusion criteria were immunosuppression, treatment for other systemic diseases, and the presence of other diseases causing nail dystrophy such as psoriasis, eczema, and lichen planus. Every patient or parent gave informed signed consent. The study was approved by our local Ethics Committee. The drug was applied once a day on the affected nail plates and nail folds for 12 weeks (end point). Patients were visited every 4 weeks. We evaluated onycholysis, nail bed discoloration, and subungueal hyperkeratosis (thickening of the subungueal region) at baseline and end point, assigning the following semiquantitative scores for onycholysis and discoloration: absence (0), 0\%-25\% (1), 25\%-50\% (2), and >50\% (3). ${ }^{14}$ For subungual hyperkeratosis, the scores were as follows: absent (0), $1 \mathrm{~mm}(1), 2 \mathrm{~mm}(2)$, and $>3 \mathrm{~mm}(3){ }^{21}$

Table I Clinical characteristics of patients

\begin{tabular}{|c|c|c|c|c|c|c|}
\hline Patient & Sex & Age (years) & Etiological agent & Affected toenails & $\begin{array}{l}\text { Duration } \\
\text { of cure (weeks) }\end{array}$ & $\begin{array}{l}\text { Affected } \\
\text { areas (\%) }\end{array}$ \\
\hline I & $\mathrm{F}$ & 40 & T. rubrum & Hallux & 4 & 20 \\
\hline 2 & $M$ & 65 & T. mentagrophytes & Halluxes & 4 & 30 \\
\hline 3 & $\mathrm{~F}$ & 25 & T. rubrum & Hallux & 12 & 60 \\
\hline 4 & $M$ & 14 & T. rubrum & Hallux + second toenail & 8 & 45 \\
\hline 5 & $M$ & 18 & T. rubrum & Halluxes & 12 & 65 \\
\hline 6 & $M$ & 40 & T. rubrum & Third toenail & 4 & 50 \\
\hline 7 & $\mathrm{~F}$ & 70 & E. floccosum & Halluxes & 8 & 35 \\
\hline 8 & $\mathrm{~F}$ & 60 & T. tonsurans & Hallux & 8 & 35 \\
\hline 9 & $\mathrm{~F}$ & 70 & T. rubrum & Halluxes + second toenail & 8 & 40 \\
\hline 10 & $M$ & 44 & T. mentagrophytes & Second toenail & 4 & 30 \\
\hline II & $M$ & 22 & T. rubrum & Hallux & 4 & 30 \\
\hline 12 & $\mathrm{~F}$ & 51 & T. rubrum & Halluxes & 8 & 35 \\
\hline 13 & $\mathrm{~F}$ & 55 & T. rubrum & Hallux + second and third toenails stoenails & 8 & 30 \\
\hline 14 & $\mathrm{~F}$ & 23 & E. floccosum & Halluxes & 8 & 25 \\
\hline 15 & $M$ & 66 & T. rubrum & Hallux & 4 & 30 \\
\hline
\end{tabular}

Abbreviations: $F$, female; $M$, male. 
We also evaluated changes of the affected area comparing baseline and end point appearance of the affected target areas, as reported..$^{22}$ Briefly, the response rate was assessed by the analysis of digital photographs of the affected nails taken at the baseline and at the end of the study. ${ }^{23}$ Data were analyzed by means of analysis of variance and Student's $t$-test for continuous data and by Mann-Whitney test for ordinal data; $P$-values $<0.05$ were considered statistically significant. Mycological cultures and potassium hydroxide stains were also performed on nail samples taken by scraping, clipping, or curetting the affected nail site and subungual region at the baseline and at the end point. Staining of fungi was performed with lactophenol cotton blue solution (SigmaAldrich, St Louis, MO, USA).

Fungistatic activity of tazarotene in vitro was investigated by disk diffusion assay (Kirby-Bauer method). ${ }^{24}$ Briefly, $1 \mathrm{~g}$ of $0.1 \%$ tazarotene gel was dissolved in $3 \mathrm{~mL}$ of physiological solution in a sterile tube. To test the effect, a concentration of $0.5 \mathrm{McFarland}$ standard was used for each fungus. For the assays, we used $0.5-5 \mathrm{~mL}$ of tazarotene solutions, respectively. The fungi analyzed were Candida albicans, C. glabrata, T. mentagrophytes, and T. verrucosum. Plates were then incubated at $28^{\circ} \mathrm{C}-30^{\circ} \mathrm{C}$ for 24 hours and 48 hours. The sensitivity was evaluated by measuring the inhibition zone diameter. All statistical analysis was performed using the statistical package SPSS16.0 (SPSS Inc, Chicago, IL, USA).

\section{Results}

Mycological cure in target nails (ie, those with mycological confirmation of onychomycosis at baseline) was observed in subungual samples collected at the first follow-up visit (4 weeks) in $40 \%$ of patients, and complete cure with complete clinical healing in all patients was observed at the end point (12 weeks). Direct microscopy and in vitro laboratory culture of the sampled material collected after 12 weeks were negative for infections. Representative clinical images of four patients at baseline and end point after tazarotene treatment are reported in Figure 1. Therapeutic results were good, with no side effects except for mild erythema on the perionychia in two patients; nail growth and tropism were also restored. As shown in Figure 2, all examined clinical parameters markedly decreased at the end point. The percentage of affected area and the proportion of affected patients decreased $(P<0.001$; Figure 2A and B). Onycholysis, subungual hyperkeratosis, and discoloration completely disappeared in all patients at the end point $(P<0.04$; Figure 2C). The nails were completely healed at the follow-up visits up to 6 months.
Patients' fungal culture test ${ }^{11}$ was performed at baseline (Figure 3); 66.7\% of patients were positive for T. rubrum (Figure 3A), $13.3 \%$ for T. mentagrophytes, $13.3 \%$ for E. floccosum, and $6.7 \%$ for T. tonsurans. Disk diffusion assay (Figure 3B and C) document the inhibition of T. mentagrophytes growth, starting with $2 \mathrm{~mL}$ of tazarotene solution, which became almost complete with $5 \mathrm{~mL}$ (Figure 3B), as well as the reduced growth of C. albicans (Figure 3C) starting with $1 \mathrm{~mL}$ of tazarotene solution. Similar results were observed with plates with $C$. glabrata and $T$. verrucosum, with greater prominence in the presence of $4 \mathrm{~mL}$ and $5 \mathrm{~mL}$ of the solution (not shown).

\section{Discussion}

In this study, we have reported the therapeutic efficacy of tazarotene $0.1 \%$ gel for the topical treatment of distal and lateral subungual onychomycosis. T. rubrum, T. mentagrophytes, and E. floccosum are the most common agents implicated in onychomycosis. ${ }^{12}$ These dermatophytes are hyaline-septated molds, whose growing fungal hyphae penetrate into the stratum corneum of the skin and nails. The fungal infection produces keratinolytic proteases, creating a gap in the living cells. ${ }^{25}$ Some fungi have developed the ability to digest keratinous debris in soil and the faculty of parasitizing keratinous tissues in animals. ${ }^{25}$ Compared to the normal skin, the nail apparatus has a different immunological profile, a strong innate immunity, and an increased expression of antimicrobial peptides. ${ }^{26}$ On the other hand, the nail apparatus has a lower level of major histocompatibility complex class-1a antigen expression, production of immunosuppressive agents, anomalies of antigen-presenting cells, and inhibition of natural killer cell activity, ${ }^{27}$ all aspects that favor onychomycosis.

Successful topical treatment of nail onychomycosis is fraught with difficulty due to several factors, including slow nail growth, the physical presence of nail plate interfering with nail bed access, and the difficulty in finding compounds with adequate pharmacologic profile for nail penetration. ${ }^{28}$ These difficulties are reflected in the high incidence of recurrence after topical therapeutic clearance. ${ }^{29}$ At present, the most frequently used topical therapies for onychomycosis are amorolfine 5\%, ciclopirox 8\% nail lacquer, and tioconazole $28 \%$ solution, which need to be applied for prolonged periods and require strong adherence by the patients. ${ }^{13}$ Amorolfine $5 \%$ nail lacquer is effective as monotherapy for the treatment of onychomycosis, but with limited efficacy when distal nail involvement is greater than $50 \%{ }^{30}$ Up to June 2014, ciclopirox was the only topical treatment approved by the 


\section{Baseline}
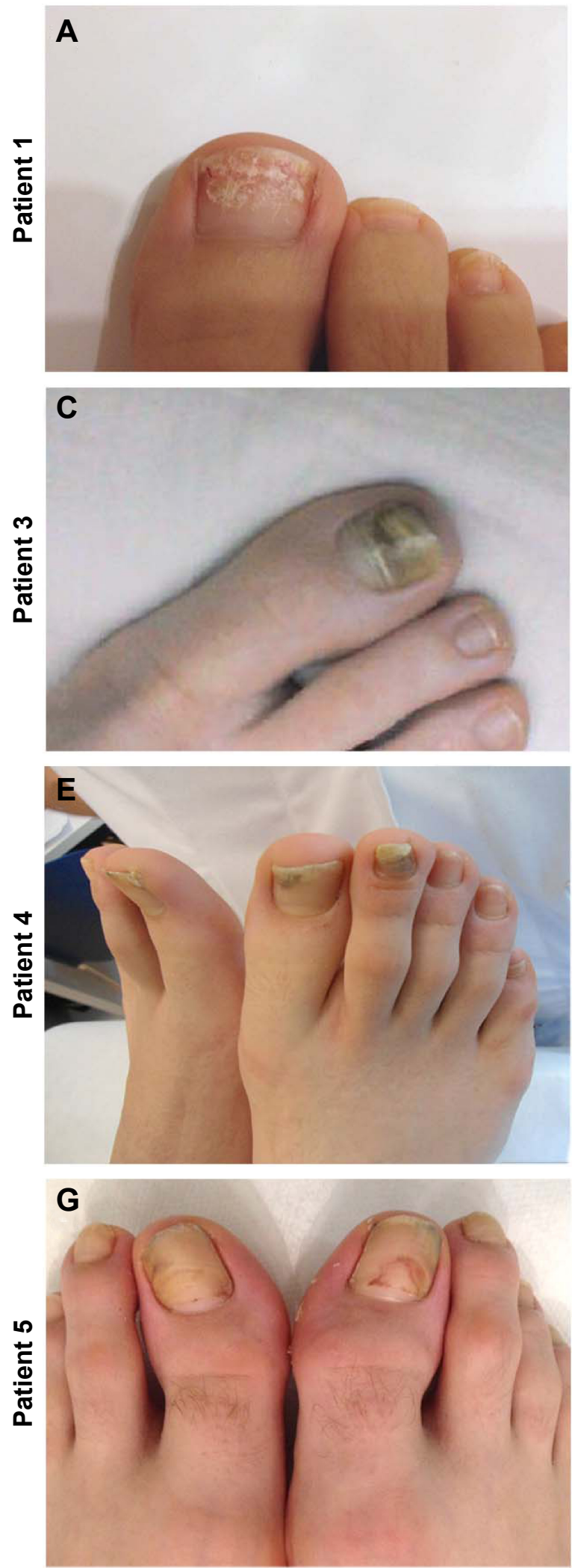

\section{End point}
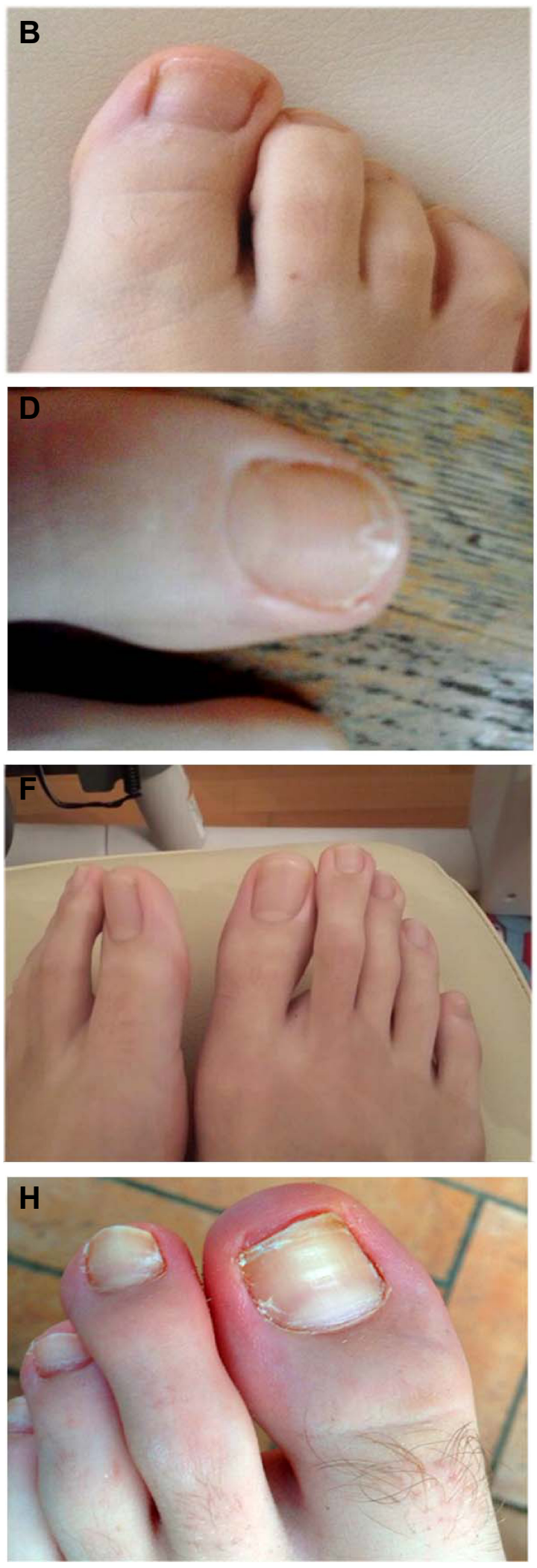

Figure I Representative clinical images of four patients at baseline and end point ( 12 weeks, topical tazarotene $0.1 \%$ gel therapy).

Notes: At baseline, patient I shows a nail involvement of $20 \%$, patient 3 of $60 \%$, patient 4 of $40 \%$ and $45 \%$ of two involved toenails, and patient 5 of $80 \%$. At the end point, all patients appear clinically cured. 
A

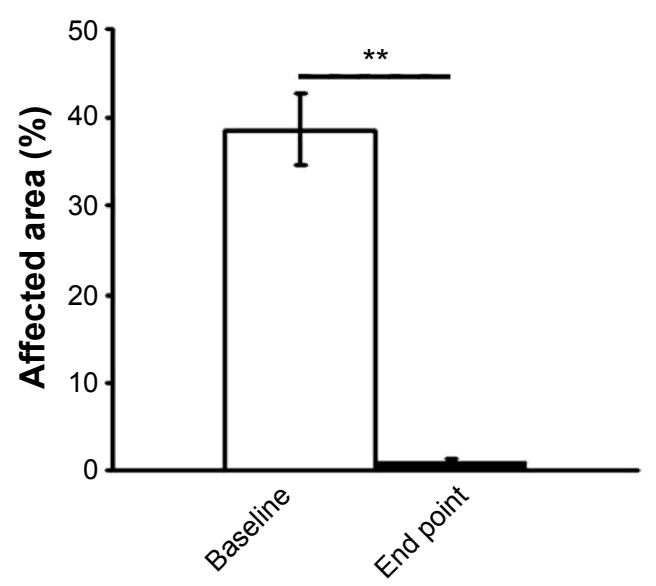

C

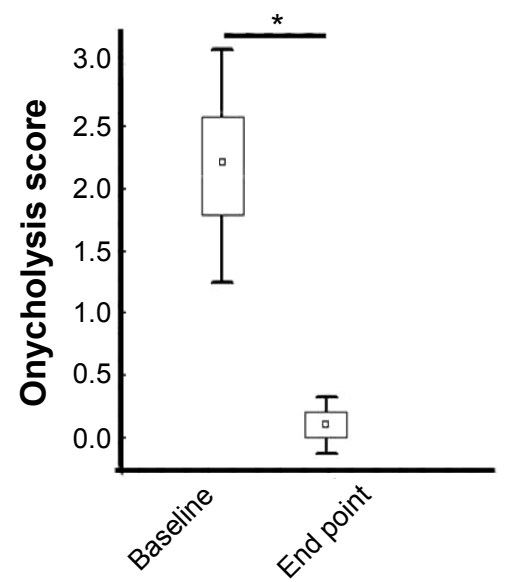

D

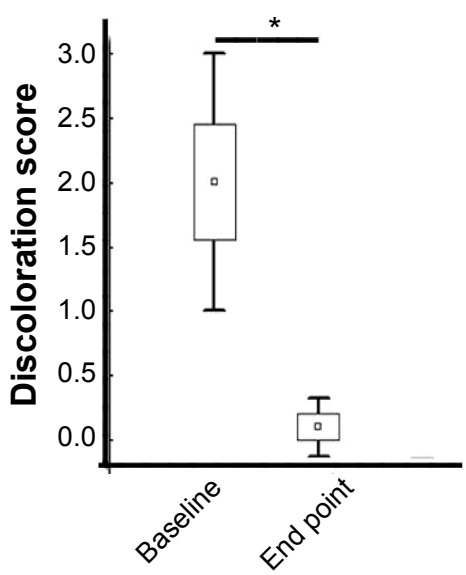

B

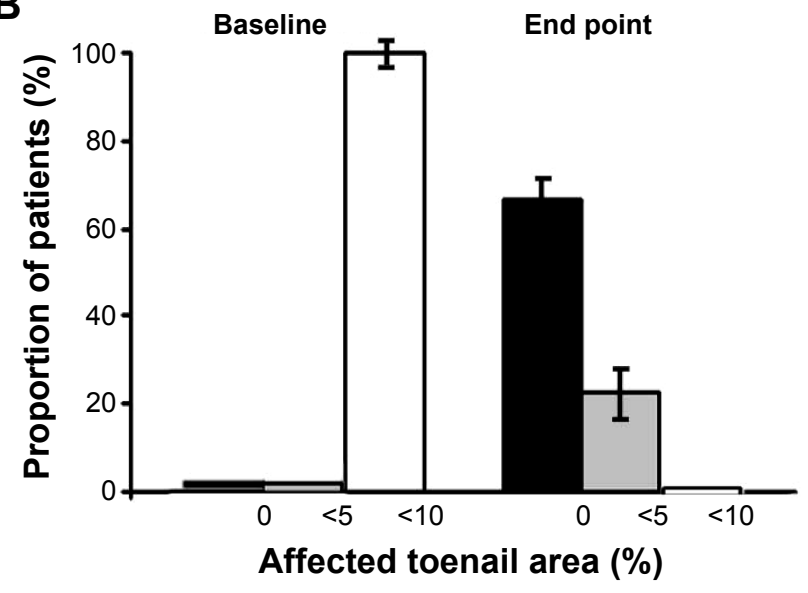

$E$

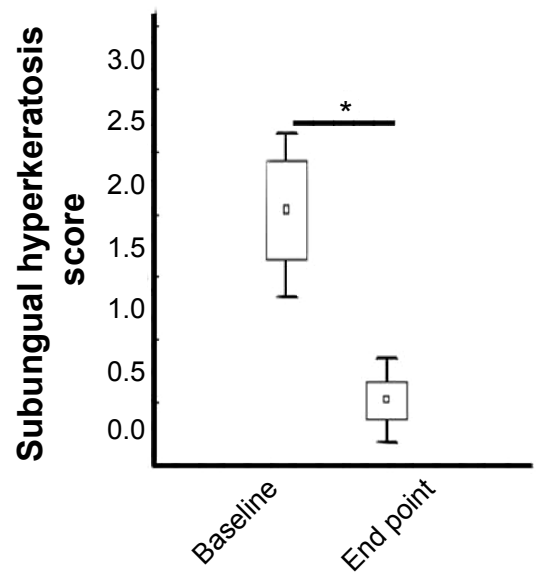

Figure 2 Semiquantitative evaluation of the clinical effect of topical treatment of tazarotene $0.1 \%$ gel.

Notes: The clinical effect of topical treatment of tazarotene $0.1 \%$ gel on onychomycosis in terms of affected area $(\mathbf{A})$ and percentage of patients $(\mathbf{B})$; $(* * P<0.00 \mathrm{I})$; box plots show the decrease of onycholysis $(\mathbf{C})$, nail bed discoloration $(\mathbf{D})$, and subungual hyperkeratosis as thickening of the subungual region (E) at the end point (I 2 weeks of topical treatment with tazarotene $0.1 \%$ gel); ${ }^{* P}<0.04$. For $(\mathbf{A})$ and $(\mathbf{B})$ error bars are representative of standard error of mean (SEM); for $(\mathbf{C}-\mathbf{E})$ error bars are representative of mean SD.

US Food and Drug Administration for onychomycosis in the US. ${ }^{28}$ The action of ciclopirox differs from that of other topical antifungal drugs, which generally act through ergosterol inhibition. The high affinity of ciclopirox for trivalent metal cations results in the inhibition of the metal-dependent enzymes responsible for the degradation of peroxides within the fungal cell. ${ }^{31}$ Efficacy of ciclopirox $8 \%$ as a topical monotherapy (ranged from $20 \%$ to $36 \%$ ) has been disappointing and limited to the mildest cases of toenail onychomycosis. ${ }^{28}$ For the remaining cases, a combined therapy with a systemic antifungal is recommended. ${ }^{28}$ Nevertheless, oral antifungals have general adverse effects, including intestinal and respiratory complaints. ${ }^{32}$ Tioconazole is an imidazole antifungal agent recommended for local infections caused by Candida ${ }^{28}$ and can be used for the topical treatment of pachyonychia. Efinaconazole $10 \%$ and tavaborole $5 \%$ solutions are new topical antifungals specifically developed for the treatment of dermatophytic onychomycosis. In Phase III clinical trials, both newer agents were applied once daily for 48 weeks without concomitant nail debridement. Reported mycologic cure rates with efinaconazole are superior to that achieved with ciclopirox $8 \%$ nail lacquer. ${ }^{33}$ In our study, complete healing after topical treatment with tazarotene was obtained at the first follow-up visit after 4 weeks in $40 \%$ of patients and complete healing in all patients after 12 weeks, with a rapid and progressive amelioration of all evaluated clinical parameters. The cure was confirmed by negative mycological culture and $\mathrm{KOH}$ stains. Sometimes, the ineffectiveness of topical treatment is due to the subungual strong hyperkeratosis, which prevents drug penetration. ${ }^{34}$ Hyperkeratosis also represents one of the most frequent features of nail psoriasis, and nail psoriatic patients also suffer from onychomycosis. ${ }^{35}$ 
A

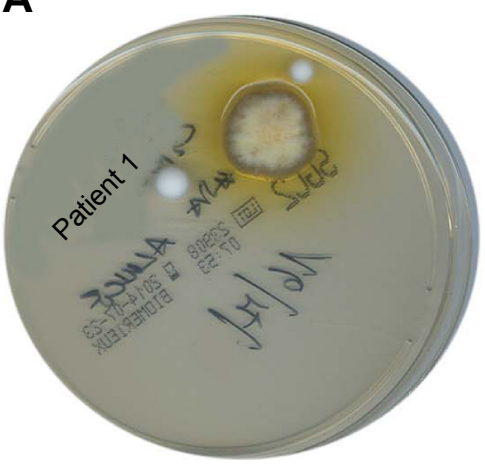

B

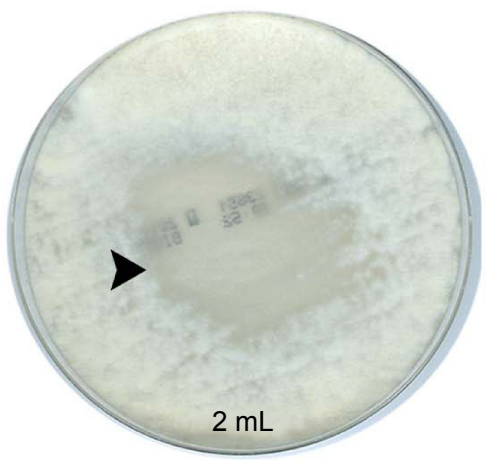

C

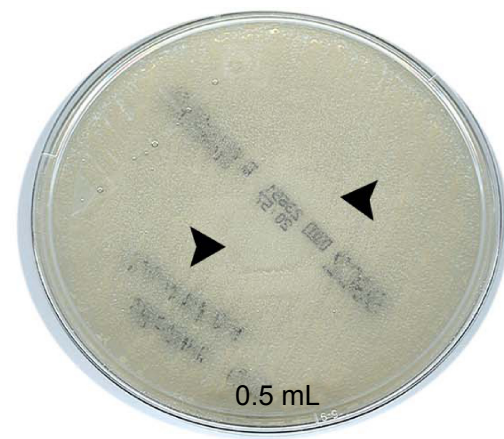

T. rubrum
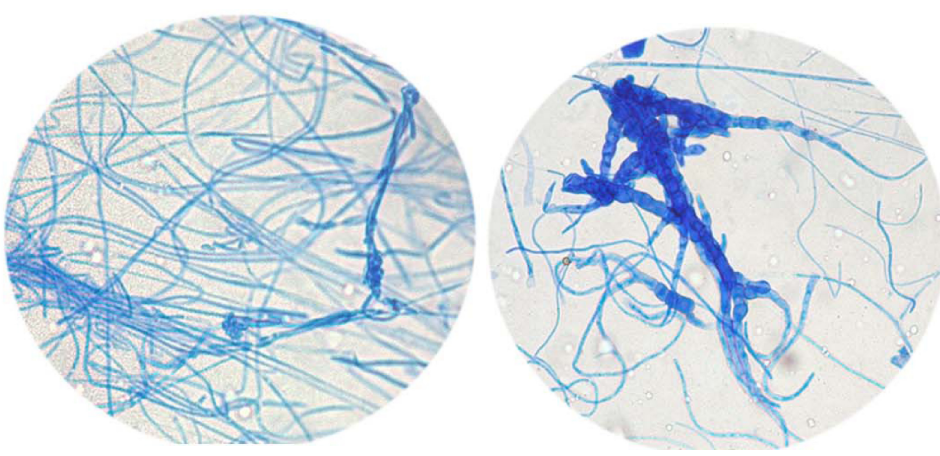

T. mentagrophytes
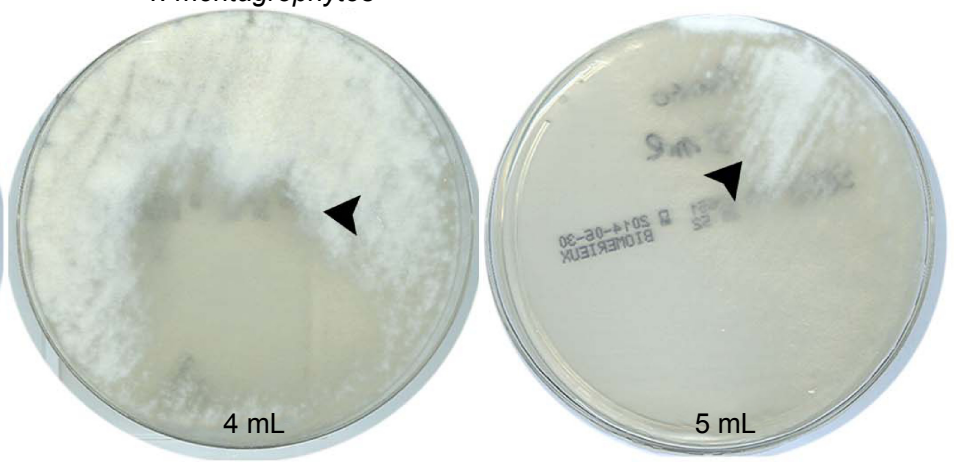

$5 \mathrm{~mL}$
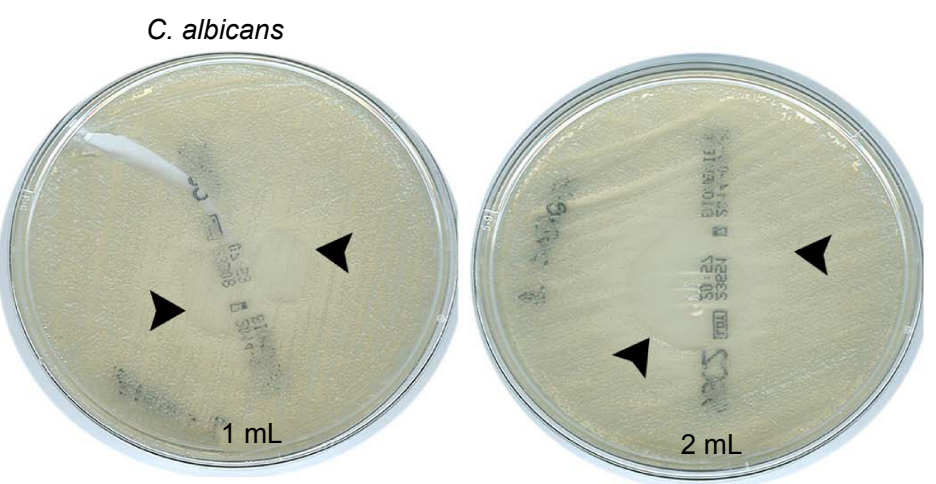

Figure 3 Patients' fungal culture test.

Notes: (A) Representative images of $T$. rubrum culture from patient I (left panel); (central and right panels) lactophenol cotton blue solution-stained images. (B and C) Disk diffusion assay of fungistatic activity of tazarotene solution. At 48 hours, the plates incubated at $28^{\circ} \mathrm{C}$ show an inhibition zone in the treated central area with different volumes of tazarotene solution.

In our patients, tazarotene has proven quite effective in reducing hyperkeratinization in fungus-infected nails. Concerning the therapeutic effects of tazarotene, our results in vitro document a direct fungistatic activity. Tazarotene is a synthetic third-generation retinoid derived from vitamin A, which has proved to be beneficial in modulating keratinocyte proliferation and in reducing inflammation. Tazarotene is used for the treatment of acne, nonmelanoma skin tumors, and inflammatory skin diseases such as psoriasis. ${ }^{36-38}$ In particular, tazarotene's effect is mediated by the intracellular upregulation of cellular retinoic acid-binding protein-II expression, ${ }^{39}$ which transactivates nuclear retinoic acid receptors (RARs), in particular RAR and RAR $\gamma$, by binding to specific DNA sequences on their promoter gene regions, leading to reduced proliferation of normal and neoplastic cells and favoring differentiation and apoptosis. ${ }^{36-38,40}$ The reduction in the excessive V-Akt murine thymoma viral oncogene homolog 1 activation supporting cell growth ${ }^{41}$ may also explain the beneficial effects of tazarotene on ungual hyperkeratosis. Moreover, tazarotene can exert a protective action on the microvascular bed against fungal infection, similarly to that reported for psoriasis patients. ${ }^{16}$ Finally, the activity of 
tazarotene against inflammation through immunomodulation may induce the blocking of fungal keratinolytic proteases, thus contributing to its efficacy. A limitation of our preliminary study results is the absence of a randomized placebo control group. Nevertheless, a recent analysis published in 2014 reveals that in 21 randomized controlled trials of dermatophytic nail onychomycosis, present in the PubMed database, placebo cure varied from $8.7 \%$ to $1.2 \%$, documenting that the pooled placebo cure mean rate is very low. ${ }^{42}$

\section{Conclusion}

Our preliminary results documented the safety and rapid efficacy of topical treatment with tazarotene on distal and lateral subungual onychomycosis, thus representing an alternative new promising therapeutic approach. Treated and cured patients were followed every 4 weeks, with no evidence of recurrence after 6 months. We also documented the fungistatic activity of tazarotene in T. mentagrophytes, T. verrucosum, C. albicans, and C. glabrata, supporting the theoretical assumption about its direct action on fungus replication. Our clinical evidence of efficacy suggests that tazarotene could be useful in those patients with treatmentresistant forms of onychomycosis or in those with evident enhanced nail thickening, wherein the therapeutic reducing action of the retinoid can be providential. Further studies concerning the specific effects of tazarotene and in a larger cohort of patients with a longer follow-up are needed to confirm the efficacy in terms of relapse and reinfection.

\section{Acknowledgments}

The authors wish to thank Denis Mariano for editing assistance.

\section{Author contributions}

All the authors have made substantial contributions to conception and design, acquisition, analysis, and interpretation of data; drafting the article and revising it; approval for the final version; and agreement for all aspects of the work related to its accuracy or integrity.

\section{Disclosure}

The authors report no conflicts of interest in this work.

\section{References}

1. Westerberg DP, Voyack MJ. Onychomycosis. Current trends in diagnosis and treatment. Am Fam Physician. 2013;88(11):762-770.

2. Kaur R, Kashyap B, Bhalla P. Onychomycosis - epidemiology, diagnosis and management. Indian J Med Microbiol. 2008;26(2):108-116.

3. Chi CC, Wang SH, Chou MC. The causative pathogens of onychomycosis in southern Taiwan. Mycoses. 2005;48(6):413-420.
4. Drake LA, Dinehart SM, Farmer ER, et al. Guidelines of care for superficial mycotic infections of the skin: tinea corporis, tinea cruris, tinea faciei, tinea manuum, and tinea pedis. Guidelines/outcomes committee. American academy of dermatology. J Am Acad Dermatol. 1996;34(2 pt 1):282-286.

5. Scher RK. Diseases of the nails. In: Conn H, editor. Current Therapy. Philadelphia, PA: The W. B. Saunders Co; 1990:736-742.

6. Tosti A, Hay R, Arenas-Guzman R. Patients at risk of onychomycosis risk factor identification and active prevention. J Eur Acad Dermatol Venereol. 2005;19(suppl 1):13-16.

7. Dias N, Santos C, Portela M, Lima N. Toenail onychomycosis in a Portuguese geriatric population. Mycopathologia. 2011;172(1):55-61.

8. Leibovici V, Hershko K, Ingber A, Westerman M, Leviatan-Strauss N, Hochberg M. Increased prevalence of onychomycosis among psoriatic patients in Israel. Acta Derm Venereol. 2008;88(1):31-33.

9. Scher RK, Tavakkol A, Sigurgeirsson B, et al. Onychomycosis: diagnosis and definition of cure. J Am Acad Dermatol. 2007;56(6):939-944.

10. Drake LA, Dinehart SM, Farmer ER, et al. Guidelines of care for superficial mycotic infections of the skin: pityriasis (tinea) versicolor. Guidelines/outcomes committee. American academy of dermatology. J Am Acad Dermatol. 1996;34(2 pt 1):287-289.

11. Scher RK. Onychomycosis: a significant medical disorder. J Am Acad Dermatol. 1996;35(3 pt 2):S2-S5.

12. Elewski BE. Onychomycosis: pathogenesis, diagnosis, and management. Clin Microbiol Rev. 1998;11(3):415-429.

13. Piraccini BM, Gianni C. Update on the management of onychomycosis. G Ital Dermatol Venereol. 2013;148(6):633-638.

14. Crawford F, Hollis S. Topical treatments for fungal infections of the skin and nails of the foot. Cochrane Database Syst Rev. 2007;18:(3) CD001434.

15. Diluvio L, Campione E, Paterno EJ, Mordenti C, El Hachem M, Chimenti S. Childhood nail psoriasis: a useful treatment with tazarotene 0.05\%. Pediatr Dermatol. 2007;24(3):332-333.

16. Bianchi L, Soda R, Diluvio L, Chimenti S. Tazarotene $0.1 \%$ gel for psoriasis of the fingernails and toenails: an open, prospective study. $\mathrm{Br}$ J Dermatol. 2003;149(1):207-209.

17. Epstein EL, Stein Gold L. Safety and efficacy of tazarotene foam for the treatment of acne vulgaris. Clin Cosmet Investig Dermatol. $2013 ; 6: 123-125$.

18. Gregoriou S, Kritsotaki E, Katoulis A, Rigopoulos D. Use of tazarotene foam for the treatment of acne vulgaris. Clin Cosmet Investig Dermatol. 2014; 7:165-170.

19. Iraci S, Bianchi L, Gatti S, Carrozzo AM, Bettini D, Nini G. Pachyonychia congenita with late onset of nail dystrophy - a new clinical entity? Clin Exp Dermatol. 1993;18(5):478-480.

20. Harris K, Hull PR, Hansen CD, et al. Transgrediens pachyonychia congenita (PC): case series of a nonclassical PC presentation. $\mathrm{Br} J$ Dermatol. 2012;166(1):124-128.

21. Klaassen KM, van de Kerkhof PC, Bastiaens MT, Plusje LG, Baran RL, Pasch MC. Scoring nail psoriasis. J Am Acad Dermatol. 2014;70(6):1061-1066.

22. Elewski BE, Rich P, Pollak R, et al. Efinaconazole $10 \%$ solution in the treatment of toenail onychomycosis: two phase III multicenter, randomized, double-blind studies. J Am Acad Dermatol. 2013;68(4):600-608.

23. Sipponen P, Sipponen A, Lohi J, Soini M, Tapanainen R, Jokinen JJ. Natural coniferous resin lacquer in treatment of toenail onychomycosis: an observational study. Mycoses. 2013;56(3):289-296.

24. Knowles RC, Moore TD. Quality control of agar diffusion susceptibility tests. Data from the quality assurance service microbiology program of the college of American pathologists. Am J Clin Pathol. 1980;74 (4 suppl):581-585.

25. Hay RJ. Fungal skin infections. Arch Dis Child. 1992;67(9): 1065-1067.

26. Dorschner RA, Lopez-Garcia B, Massie J, Kim C, Gallo RL. Innate immune defense of the nail unit by antimicrobial peptides. $\mathrm{J}$ Am Acad Dermatol. 2004;50(3):343-348. 
27. Ito T, Meyer KC, Ito N, Paus R. Immune privilege and the skin. Curr Dir Autoimmun. 2008;10:27-52.

28. Del Rosso JQ. The role of topical antifungal therapy for onychomycosis and the emergence of newer agents. $J$ Clin Aesthet Dermatol. 2014; 7(7): $10-18$

29. De Cuyper C, Hindryckx PH. Long-term outcomes in the treatment of toenail onychomycosis. Br J Dermatol. 1999;141(suppl 56):15-20.

30. Chang $\mathrm{CH}$, Young-Xu Y, Kurth T, Orav JE, Chan AK. The safety of oral antifungal treatments for superficial dermatophytosis and onychomycosis: a meta-analysis. Am J Med. 2007;120(9):791-798.

31. Subissi A, Monti D, Togni G, Mailland F. Ciclopirox: recent nonclinical and clinical data relevant to its use as a topical antimycotic agent. Drugs. 2010;70(16):2133-2152.

32. Epstein E. How often does oral treatment of toenail onychomycosis produce a disease-free nail? An analysis of published data. Arch Dermatol. 1998;134(12):1551-1554.

33. Gupta AK, Paquet M. Efinaconazole $10 \%$ nail solution: a new topical treatment with broad antifungal activity for onychomycosis monotherapy. J Cutan Med Surg. 2014;18(3):151-155.

34. Dias MF, Quaresma-Santos MV, Bernardes-Filho F, Amorim AG, Schechtman RC, Azulay DR. Update on therapy for superficial mycoses: review article part I. An Bras Dermatol. 2013;88(5):764-774.

35. Klaassen KM, Dulak MG, van de Kerkhof PC, Pasch MC. The prevalence of onychomycosis in psoriatic patients: a systematic review. J Eur Acad Dermatol Venereol. 2014;28(5):533-541.
36. Bianchi L, Orlandi A, Campione E, et al. Topical treatment of basal cell carcinoma with tazarotene: a clinicopathological study on a large series of cases. Br J Dermatol. 2004;151(1):148-156.

37. Thacher SM, Vasudevan J, Chandraratna RA. Therapeutic applications for ligands of retinoid receptors. Curr Pharma Des. 2000;6(1):25-58.

38. Orlandi A, Bianchi L, Costanzo A, Campione E, Giusto Spagnoli L, Chimenti S. Evidence of increased apoptosis and reduced proliferation in basal cell carcinomas treated with tazarotene. J Invest Dermatol. 2004;122(4):1037-1041.

39. Bushue N, Wan YJ. Retinoid pathway and cancer therapeutics. $A d v$ Drug Deliv Rev. 2010;62(13):1285-1298.

40. Lotan R. Retinoids in cancer chemoprevention. FASEB J. 1996;10(9): 1031-1039.

41. Cervelli V, Scioli MG, Gentile P, et al. Platelet-rich plasma greatly potentiates insulin-induced adipogenic differentiation of human adipose-derived stem cells through a serine/threonine kinase Aktdependent mechanism and promotes clinical fat graft maintenance. Stem Cells Transl Med. 2012;1(3):206-220.

42. Gupta AK, Daigle D, Paquet M. Therapies for onychomycosis: a systematic review and network meta-analysis of mycological cure. J Am Podiatr Medl Assoc. Epub 2014 Jul 17.
Drug Design, Development and Therapy

\section{Publish your work in this journal}

Drug Design, Development and Therapy is an international, peerreviewed open-access journal that spans the spectrum of drug design and development through to clinical applications. Clinical outcomes, patient safety, and programs for the development and effective, safe, and sustained use of medicines are a feature of the journal, which

\section{Dovepress}

has also been accepted for indexing on PubMed Central. The manuscript management system is completely online and includes a very quick and fair peer-review system, which is all easy to use. Visit http://www.dovepress.com/testimonials.php to read real quotes from published authors.

Submit your manuscript here: http://www.dovepress.com/drug-design-development-and-therapy-journal 